\title{
Efeitos da vergonha em sintomas depressivos em pessoas com e sem doença física crónica: Os papéis mediadores da autocompaixão e da ação comprometida
}

\author{
The effect of shame on depressive symptoms in people with and without chronic physical \\ disease: Exploring the mediator role of self-compassion and committed action
}

\author{
Daniela Veiga (1) \\ Inês A. Trindade (1) \\ Cláudia Ferreira (1)
}

(1) CINEICC - Center for Research in Neuropsychology and Cognitive and Behavioral Intervention, Faculdade de Psicologia e de Ciências da Educação, Universidade de Coimbra, Coimbra, Portugal.

\section{Resumo}

Objetivo: O presente estudo teve como objetivo testar o potencial efeito mediador da autocompaixão e da ação comprometida na relação entre vergonha e sintomatologia depressiva, em pessoas sem e com diagnóstico de doença física crónica. Adicionalmente, foram exploradas as diferenças em relação a essas variáveis entre os dois grupos. Métodos: $A$ amostra foi constituída por 453 participantes (223 com e 230 sem diagnóstico de doença física crónica), os quais responderam numa plataforma online a um protocolo de medidas de autorrelato de vergonha, autocompaixão, ação comprometida e sintomas depressivos. Resultados: Os participantes com diagnóstico de doença crónica apresentaram níveis significativamente $(p<0,05)$ superiores de vergonha e de sintomatologia depressiva, e níveis inferiores de ação comprometida, comparativamente aos participantes sem doença física crónica. Contudo, não foram encontradas diferenças significativas entre os dois grupos relativamente às competências autocompassivas. As análises de correlação revelaram que a vergonha se associa negativamente à autocompaixão e ação comprometida e positivamente á sintomatologia depressiva, tanto no grupo sem como no grupo com diagnóstico de doença física crónica. Os resultados da path analysis indicaram que sentimentos de vergonha têm um impacto significativo, explicando $41 \%$ da variância da sintomatologia depressiva, parcialmente via menores níveis de autocompaixão e de ação comprometida. Os resultados da análise multigrupos demonstraram que o modelo testado é plausível nos dois grupos em estudo. Conclusões: Este estudo parece fornecer importantes contributos para a compreensão do impacto protetor das competências autocompassivas e da adoção de ações comprometidas para a saúde mental, tanto para pessoas sem como com diagnóstico de doença física crónica. De facto, os resultados sugerem que estes processos de regulação emocional são importantes mecanismos mediadores da relação entre vergonha e sintomas depressivos. Finalmente, estes dados parecem suportar o desenvolvimento de abordagens mais eficazes para a promoção da saúde psicológica para pessoas sem e com doença crónica.

Palavras-Chave: Autocompaixão; Ação comprometida; Doença crónica; Sintomatologia depressiva; Vergonha.

\section{DI\&D | ISMT}

rpics@ismt.pt

https://rpics.ismt.pt
Publicação em Acesso Aberto

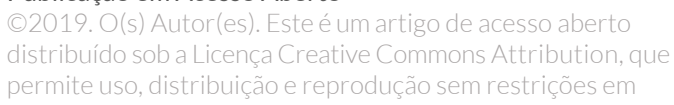

qualquer meio, desde que o trabalho original seja devidamente

citado.
Cláudia Ferreira

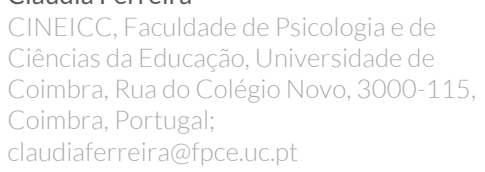




\begin{abstract}
Aim: The current study aimed at examining the potential mediator role of self-compassion and committed action on the link between shame and depressive symptomatology in people with and without a diagnosis of chronic disease. Additionally, differences between these two groups were explored regarding these variables. Method: The sample included 453 participants (223 with chronic disease and 230 without a diagnosis of chronic disease), who completed an online survey. Results: Participants with a diagnosis of chronic disease significantly $(p<0,05)$ presented higher levels of shame feelings and depressive symptoms, and lower levels of committed action than participants without a chronic disease. Nonetheless, no significant differences in self-compassion abilities were found between the groups. Correlational results revealed that shame was negatively associated with self-compassion and committed action, and positively with depressive symptomatology, in both groups. Path analysis results indicated that shame feelings have a significant impact on depressive symptomatology (explaining $41 \%$ of its variance), mediated through decreased self-compassion and committed action. A multi-group analysis revealed that this model was invariant between study groups. Conclusions: This study seems to provide important contributions to understand the protective impact of self-compassionate abilities and the adoption in committed action in mental health, both for people with or without a chronic disease diagnosis. Indeed, these processes appear to be important mediating mechanisms that seem to minimize the negative effects of shame on depressive symptomatology. These findings seem to support the development of more effective approaches for the promotion of psychological health for people with or without a chronic disease diagnosis.
\end{abstract}

Keywords: Committed action; Chronic disease; Depressive symptomatology; Shame; Self-compassion.

\title{
Introdução
}

Vasta literatura tem vindo a enfatizar o papel da autocompaixão enquanto estratégia de regulação emocional particularmente útil, sublinhando o seu papel protetor na saúde mental (e.g., associando-se à diminuição dos sintomas psicopatológicos) e qualidade de vida, tanto em amostras de doentes crónicos (Pinto-Gouveia, Duarte, Matos, \& Fráguas, 2013; Sirois \& Hirsch, 2019; Sirois \& Rowse, 2016) como em amostras da população geral (e.g., Körner et al., 2015; Neff, 2003b). A autocompaixão pode ser compreendida como a capacidade do indivíduo de ser compreensivo e caloroso relativamente a experiências e aspetos do eu percecionados como indesejáveis ou negativos, os quais são percecionados como parte da experiência humana comum e numa atitude mindful (Neff, 2003b). Esta estratégia de regulação emocional adaptativa não envolve o evitamento de insucessos, inadequações e falhas pessoais, nem implica autocriticismo e julgamentos severos; mas sim uma atitude de aceitação, bondade e compreensão perante contextos de dor e sofrimento (e.g., Folkman \& Moskowitz, 2000; Neff, 2003a). Vários estudos apontam que maiores níveis de autocompaixão se associam a menores níveis de ansiedade, ruminação e sintomas depressivos (e.g., Costa \& Pinto-Gouveia, 2011; Körner et al., 2015; MacBeth \& Gumley, 2012; Neff, 2003a, 2003b, 2009). Por outro lado, a literatura parece demonstrar que menores níveis de autocompaixão constituem um fator de vulnerabilidade para o desenvolvimento e manutenção de sintomas psicopatológicos, nomeadamente de sintomatologia depressiva (e.g., Körner et al., 2015; Krieger, Altenstein, Baetting, Doering, \& Holtforth, 2013). Ainda, em estudos com amostras de doentes crónicos, a autocompaixão é apontada como tendo um papel fundamental na promoção de comportamentos de saúde (Sirois \& Hirsch, 2019; Sirois \& Rowse, 2016).

Adicionalmente, a literatura parece demonstrar que competências autocompassivas podem ser um antídoto face ao impacto negativo das experiências de vergonha no bem-estar psicológico dos indivíduos (e.g., Benda, Kadlečík, \& Loskotová, 2018; Ferreira, Pinto-Gouveia, \& Duarte, 2013; Gilbert \& Procter, 
2006; Zhang et al., 2018). Embora esta relação entre autocompaixão e vergonha esteja bem documentada para diversas amostras (e.g., população geral, doentes depressivos), em amostras de doentes crónicos encontra-se escassamente estudada. Contudo, um estudo recente com uma amostra de doentes com doença inflamatória intestinal de Trindade, Ferreira e Pinto-Gouveia (2017a) demonstra que a vergonha associada à doença crónica (tal como à sua sintomatologia ou consequências dos tratamentos) apresenta uma associação negativa com competências autocompassivas.

A vergonha tem sido definida como uma emoção dolorosa a qual funciona como uma resposta defensiva ou como um sinal de alerta perante o risco de ser criticado, diminuído e excluído pelo grupo social (Gilbert, 1998, 2002). Gilbert $(1998,2007)$ sugere que existem duas dimensões na experiência de vergonha: a vergonha externa, cujo foco de atenção se prende com a perceção de que se existe negativamente na mente do outro (i.e., a perceção que os outros o veem como inadequado, defeituoso, inferior e indesejado); e a vergonha interna que se relaciona com a perceção negativa face a atributos e comportamentos pessoais (i.e., associa-se a experiências de autodesvalorização, inadequação, isolamento e vazio). Estes dois tipos de vergonha ativam uma série de respostas defensivas (e.g., submissão na vergonha externa e autojulgamento na vergonha interna) com o objetivo de esconder ou corrigir características e atitudes avaliadas como negativas e, assim, se proteger da crítica e/ou a rejeição dos outros (Gilbert, 1998, 2002; Gilbert \& Irons, 2005, 2009). No entanto, vários estudos têm demonstrado o efeito paradoxal da vergonha, evidenciando a associação entre elevados níveis de vergonha e uma vasta de sintomas psicopatológicos, nomeadamente sintomatologia depressiva (e.g., Gilbert, 2000; Kim, Thibodeau, \& Jorgensen, 2011; Pinto-Gouveia \& Matos, 2011; Trindade, MartaSimões, Ferreira, \& Pinto-Gouveia, 2018a).

Especificamente, os indivíduos com doença crónica parecem apresentar uma maior propensão a experienciar sentimentos de vergonha, associados a experiências relacionadas à doença e sua sintomatologia ou consequências dos tratamentos (e.g., Casati, Toner, De Rooy, Drossman, \& Maunder, 2000; Trindade, Ferreira, \& Pinto-Gouveia, 2017b; Trindade et al., 2018a). Adicionalmente, investigação prévia com doentes crónicos tem demonstrado a relevância desta emoção, salientando o seu impacto negativo no funcionamento e bem-estar psicológico dos indivíduos (e.g., Trindade et al., 2017a, 2017b, 2018a; Trindade, Duarte, Ferreira, Coutinho, \& Pinto-Gouveia, 2018). Embora estudos em amostras da população geral tenham vindo a demonstrar a forte relação entre sentimentos gerais de vergonha (i.e., não específicos em relação a conteúdo, por exemplo relacionado com uma doença) e diversos indicadores de psicopatologia (e.g., Kim et al., 2011; Matos \& Pinto-Gouveia, 2010; Pinto-Gouveia \& Matos, 2011) e a menores níveis de ação comprometida (Trindade, Marta-Simões, Ferreira, \& Pinto-Gouveia, 2018b), o estudo acerca da relação entre ação comprometida e sentimentos gerais de vergonha estão claramente menos explorados na população com doença crónica.

A ação comprometida é considerada um dos processos chave do modelo de flexibilidade psicológica da Terapia de Aceitação e Compromisso e caracteriza-se pela habilidade em assumir uma gama ampla de ações efetivas, guiadas pelos valores de vida (Hayes, Luoma, Bond, Masuda, \& Lillis, 2006). Esses comportamentos ativamente escolhidos (Hayes et al., 2006; Hayes, Pistorello, \& Levin, 2012) são caracterizados como flexíveis e persistentes, capazes de incorporar angústia, dor, fracasso e desconforto que possam estar implicados no processo de busca de valores (McCracken, 2013). Embora tenha sido pouco investigada, particularmente em amostras específicas (e.g., em doentes crónicos), alguns estudos 
exploraram os possíveis efeitos positivos de agir de acordo com os valores de vida escolhidos como preditor de saúde mental e física, tanto em amostras da população geral como em amostras clínicas (Galán et al., 2019; McCracken, 2013; McCracken, Chilcot, \& Norton, 2014; Michelson, Lee, Orsillo, \& Roemer, 2011; Trindade et al., 2018b; Trompetter et al., 2013). A ação comprometida parece atenuar o efeito da vergonha relacionada à doença em relação a piores indicadores de saúde mental (Trindade et al., 2017b; Trindade et al., 2018b). De facto, dados empíricos recentes, num grupo de pessoas sem doença crónica e num grupo de mulheres com cancro da mama, parecem indicar a associação negativa da ação comprometida com a vergonha e sintomas depressivos, o que parece demonstrar que permanecer em ações guiadas pelos valores pessoais tem um efeito protetor no impacto da vergonha e na manifestação de sintomas depressivos (Trindade et al., 2018b).

Em suma, a população com doença física crónica, devido a maior propensão para a vivência de experiências de vergonha (e.g., Casati et al., 2000; Trindade et al., 2017b, 2018a) e a maior sintomatologia depressiva reportada (Pinto-Gouveia et al., 2013), parece ser uma população em que os processos de autocompaixão e ação comprometida poderão assumir um papel particularmente importante para a promoção da sua saúde mental.

Dada a escassez de estudos comparativos entre população com e sem doença física crónica, um dos objetivos deste trabalho foi testar diferenças entre sentimentos de vergonha, autocompaixão, ação comprometida e sintomatologia depressiva entre dois grupos. Adicionalmente, este estudo teve como objetivo principal testar o potencial papel mediador da autocompaixão e ação comprometida na relação entre vergonha e sintomas depressivos. Foi hipotetizado que o efeito negativo da vergonha nos sintomas depressivos é mediado por menores níveis de autocompaixão e de comprometimento com ações congruentes com valores de vida. Hipotetizámos, ainda, que estas relações são universais (Coutinho, Trindade, \& Ferreira, 2019; Trindade et al., 2018b), esperando-se que o modelo testado seja invariante nos dois grupos em estudo.

\section{Método}

\section{Participantes}

A amostra deste estudo foi composta por 453 participantes adultos, divididos em dois grupos: grupo com diagnóstico de doença física crónica $(n=223)$ e grupo sem diagnóstico de doença física crónica $(n=230)$. O grupo com doença crónica, com idades compreendidas entre os 18 e 65 anos ( $M=38,53 ; D P=11,82)$, inclui 196 mulheres e 27 homens. Em relação à escolaridade, este grupo apresentou uma média de 13,97 ( $D P$ = 2.88) anos de escolaridade. Dos participantes sem doença crónica, 160 eram mulheres e 70 homens, com idades a variar entre os 18 e 62 anos $(M=31,30 ; D P=10,51)$, com uma média de 14,28 $(D P=2,60)$ anos de escolaridade. Os dois grupos apresentaram diferenças significativas em relação à idade $(t(451)=-6,89 ; p<$ 0,001), com o grupo com doença crónica a apresentar uma idade média superior comparativamente ao grupo sem doença crónica, e ao género $\left(\chi^{2}(1)=22,60, p<0,001\right)$, com o grupo com doença crónica a apresentar menos homens e mais mulheres em relação ao grupo sem doença crónica. Além disso, no que respeita ao estado civil, também foram encontradas diferenças significativas em ambos os grupos $\left(\chi^{2}(4)=\right.$ $22,80, p<0,001)$, com a maioria dos participantes do grupo com doença crónica casados (51,1\%), enquanto 
que no grupo sem doença crónica a maioria era solteira (60,9\%). Em relação aos anos de escolaridade não foram encontradas diferenças significativas em ambos os grupos $(t(451)=1,22 ; p=0,224)$.

Dos 223 participantes do grupo com doença crónica, 173 (77,6\%) reportaram ter apenas uma doença crónica e 50 (22.4\%) participantes reportaram ter diagnóstico de duas ou mais doenças crónicas. Das 74 doenças crónicas presentes na amostra, as cinco doenças mais prevalentes neste grupo foram lúpus (33,63\%), fibromialgia (10,76\%), endometriose (9,87\%), asma (8,07\%) e síndrome de Sjorgen (6,28\%). A Tabela 1 apresenta a frequência de doença crónica, definida a partir de cada um dos sistemas do organismo humano envolvidos, reportada pelos participantes do grupo com diagnóstico de doença física crónica.

\section{Tabela 1}

Caracterização do Grupo de Participantes com Doença Crónica por Sistema $(n=223)$

\begin{tabular}{lcc}
\hline Sistema & $n$ & $\%$ \\
\hline Reumatológicas e musculoesqueléticas & 162 & 72,64 \\
Respiratórias & 32 & 14,35 \\
Endócrinas e hematológicas & 27 & 12,11 \\
Ginecológicas & 23 & 10,31 \\
Renais & 18 & 8,07 \\
Gastrointestinais & 17 & 7,62 \\
Dermatológicas & 12 & 5,38 \\
Cardiovasculares & 9 & 4,04 \\
Neoplásicas & 3 & 1,35 \\
Neurológicas & 3 \\
Neuromusculares & 2 & 0,90 \\
Oftalmológicas & 2 & 0,90
\end{tabular}

\section{Medidas}

Escala de Vergonha Externa e Interna (EISS); Ferreira, Moura-Ramos, Matos, \& Galhardo (2018). A EISS avalia numa única medida de experiência de vergonha externa e interna. Esta escala é composta por oito itens, gerados para medir os quatro domínios centrais da experiência de vergonha, e presentes quer na vergonha externa quanto interna: inferioridade/inadequação, senso de exclusão, inutilidade/vazio e crítica/julgamento. Para avaliar cada um destes domínios existe um item, num total de quatro itens para uma dimensão externa (e.g., "Sinto que os outros me veem como desinteressante") e dimensão interna da vergonha (e.g., "Sinto que sou diferente e inferior aos outros"). Os participantes devem responder a cada item através de uma escala de 5 pontos ( 0 = "Nunca" a 4 = "Sempre"). As pontuações variam entre 0 e 32 pontos, sendo os valores mais elevados indicadores de maiores níveis de vergonha. No presente estudo foi unicamente usada a pontuação total da escala. A versão original da escala apresenta boas qualidades psicométricas, com os valores de alfas de Cronbach para a escala total de 0,89, respetivamente (Ferreira et al., 2018).

Escala de Auto-Compaixão (Self-Compassion Scale (SELFCS); Neff (2003b); versão portuguesa de Castilho \& Pinto-Gouveia (2011)]. Esta medida de autorresposta, constituída por 26 itens, visa avaliar a autocompaixão, através de seis dimensões: subescalas Calor/Compreensão (e.g., "Sou tolerante com os meus erros e 
inadequações"); Humanidade Comum (e.g., "Tento ver os meus erros e falhas como parte da condição humana"); Mindfulness (e.g., "Quando alguma coisa dolorosa acontece tento ter uma visão equilibrada da situação"); Autojulgamento (e.g., "Desaprovo-me e faço julgamentos acerca dos meus erros e inadequações”), o Isolamento (e.g., "Quando falho nalguma coisa importante para mim tendo a sentir-me sozinho(a) no meu fracasso") e Sobre-Identificação (e.g., "Quando alguma coisa dolorosa acontece tendo a exagerar a sua importância"). A resposta aos itens é realizada através de uma escala de Likert de 5 pontos ( 1 = "quase nunca" a 5 = "quase sempre"). Os valores de consistência interna revelaram-se bons, tanto na versão original como na versão portuguesa ( $\alpha$ de Cronbach = 0,92 e 0,89, respetivamente) (Castilho \& Pinto-Gouveia, 2011; Neff, 2003b). No estudo foi utilizado o compósito de autocompaixão, constituído pelas três dimensões positivas desta medida. Relativamente ao compósito de autocompaixão as pontuações variam entre 13 a 65 pontos, traduzindo os valores mais altos níveis superiores de autocompaixão.

Questionário de Ação Comprometida [Commited Action Questionnaire (CAQ-8); McCracken et al. (2014); versão portuguesa de Trindade et al. (2018b)]. O CAQ-8 é um instrumento de autorresposta, constituído por 8 itens, que avalia a ação comprometida. Este questionário é composto por uma subescala positiva (e.g., "Prefiro mudar a forma de lidar com um objetivo do que desistir") e uma subescala negativa (e.g., "Acho difícil continuar uma atividade a não ser que sinta que é bem-sucedida"), cada uma com 4 itens. É solicitado aos participantes que respondam numa escala de 7 pontos a cada item $(0=$ "Nunca verdadeiro" a $6=$ "Sempre verdadeiro"). No presente estudo foi utilizada a pontuação total da escala, a qual pode variar entre 0 a 48 pontos. Valores mais altos nesta medida revelam maior adoção de ação comprometida. No que respeita à consistência interna, esta medida apresentou alfas de Cronbach de 0,87 na versão original (McCracken et al., 2014) e de 0,86 e de 0,79 na versão portuguesa, num grupo de pessoas sem doença crónica e num grupo de mulheres com carcinoma da mama, respetivamente (Trindade et al., 2018b).

Escalas de Ansiedade, Depressão e Stress [Depression Anxiety Stress Scale (DASS-21); Lovibond \& Lovibond (1995); versão portuguesa de Pais-Ribeiro, Honrado, \& Leal (2004)]. A DASS-21 é uma medida de autorresposta que avalia sintomas emocionais negativos, através de três subescalas: depressão, ansiedade e stress, com sete itens cada. É solicitado aos sujeitos que respondam se a cada afirmação de acordo com a sua ocorrência durante a semana anterior, numa escala tipo Likert de 4 pontos ( 0 = "Não se aplicou nada a mim" a 3 = "Aplicou-se a mim a maior parte das vezes"). No presente estudo, apenas a subescala de depressão foi considerada, a qual pode compreender valores entre 0 a 21 (correspondendo valores mais elevados a maior severidade da sintomatologia depressiva). Na versão original, a consistência interna nesta subescala foi de 0,88 (Lovibond \& Lovibond, 1995) e de 0,85 na versão portuguesa (Pais-Ribeiro et al., 2004).

Os valores de consistência interna no presente estudo de todas as escalas mencionadas encontram-se descritas na Tabela 2.

\section{Procedimento}

O presente estudo faz parte integrante de um projeto I\&D sobre os diferentes processos de regulação emocional e qualidade de vida, em amostras de indivíduos com diagnóstico de doença física crónica. Todos os requisitos éticos e deontológicos inerentes à investigação científica em Psicologia foram respeitados, nomeadamente na recolha da amostra e no tratamento de dados.

Depois da obtenção de autorização (com o número 13/2016) por parte da Comissão de Ética da instituição de ensino superior envolvida, procedeu-se à recolha de amostra através da rede social Facebook e por via e-mail, 
através do método de amostragem exponencial não-discriminante de bola-de-neve. Simultaneamente, foram contactadas 32 Associações Portuguesas de doentes com doença crónica e dessas sete aceitaram colaborar (e.g., Associação Portuguesa de Apoio a Mulheres com Endometriose, Associação Nacional de Fibrose Quística, Associação Portuguesa de Insuficientes Renais), divulgando o estudo entre os seus membros registados como doentes (através de listas de dados pré-existentes) ou através de redes sociais. O convite para a participação no estudo incluía um link que direcionava os participantes para a versão online do questionário, bem como para informações sobre o objetivo, procedimentos e critérios de inclusão na investigação (pessoas com idades compreendidas entre os 18 e 65 anos de idade). A todos os participantes foi solicitado que autorizassem a sua colaboração no estudo através de consentimento informado, e foi assegurado o caráter voluntário da sua participação, bem como o anonimato e confidencialidade dos dados obtidos.

Os questionários de autorresposta continham uma primeira parte de recolha de dados biográficos, na qual se questionava a existência de um (ou mais) diagnóstico(s) prévio(s) de doença física crónica. No caso de a resposta a esta questão ser afirmativa, os participantes eram direcionados para um conjunto de questões abertas relacionadas com a caracterização e com o impacto da doença (e.g., identificação do diagnóstico, tempo desde o diagnóstico, tipo de tratamento).

A amostra inicial foi constituída por 471 participantes ( 239 com doença física crónica e 232 sem diagnóstico de doença física crónica), com idades compreendidas entre os 17 e os 79 anos. Contudo, foram excluídos 18 participantes desta amostra: 16 participantes que integravam o grupo de doentes crónicos (14 devido ao critério da idade, i.e., por indicarem idades superiores a 65 anos; e os restantes dois por uma resposta inespecífica em relação à identificação da doença crónica e um por reportar doença psiquiátrica, i.e., perturbação bipolar) e dois participantes do grupo de pessoas sem diagnóstico de doença crónica, um por reportar uma idade inferior a 18 anos e outro por ter afirmado não aceitar participar na investigação, após leitura do consentimento informado.

\section{Estratégia Analítica}

A análise de dados foi realizada com recurso ao software IBM SPSS Statistics 22.0 do SPSS (SPSS IBM) e ao AMOS 22 (Arbuckle, 2013).

Foram realizadas análises estatísticas descritivas (médias e desvios-padrão), de forma a explorar as características dos grupos nas variáveis em estudo. Para explorar as diferenças entre os grupos em relação às variáveis estudadas, foram realizadas análises independentes do teste $t$ de Student. Seguidamente, foram efetuadas análises de correlação de Pearson de forma a compreender as associações entre os diversos constructos da presente investigação. As magnitudes dos resultados foram discutidas com base nas recomendações de Cohen, considerando-se de magnitude fraca correlações inferiores a 0,30, moderada entre 0,30 e 0,49 e magnitudes fortes iguais ou superiores a 0,50, para um nível de significância de 0,05 (Cohen, Cohen, West, \& Aiken, 2003).

Através do software AMOS 22 foram conduzidas path analysis, para testar um modelo de mediação no qual se hipotetiza que o efeito da vergonha (EISS; variável exógena) na sintomatologia depressiva (DASS-21-DEP; variável endógena) é mediado pelo efeito mediador da autocompaixão e da ação comprometida (SELFC-AC; CAQ-8; variáveis endógenas mediadoras) (Kline, 2005). Para testar a significância dos coeficientes da path e para calcular o modelo estatístico foi utilizado o método de Máxima Verossimilhança. Além disso, foram utilizados uma série de testes que visam calcular a qualidade do ajustamento do modelo: o Qui-Quadrado $\left(\chi^{2}\right)$, 
que revela um bom ajustamento do modelo quando é não significativo; o Índice de Ajustamento Comparativo (Comparative Fit Index; CFI) e o Índice de Tucker Lewis (Tucker Lewis Index; TLI), cujos valores superiores a 0,95 são considerados como evidência de um bom ajuste; e Raiz do Erro Quadrático Médio de Aproximação (Root-Mean Square Error of Approximation; RMSEA) cujos valores inferiores a 0,06 indicam um bom ajustamento (Hu \& Bentler, 1999). A partir de bootstrapping (com 5000 amostras) foram testados os efeitos diretos, indiretos e totais estabelecidos entre as variáveis, com intervalo de confiança (IC) corrigido de $95 \%$ (bias-corrected confidence intervals). Nesta análise, quando o intervalo de confiança não inclui o zero os efeitos são considerados estatisticamente significativos (Kline, 2005).

Por fim, com o objetivo de verificar se existem diferenças significativas no modelo final testado entre os dois grupos em comparação, foi realizada uma análise multigrupos. Em primeiro, com o objetivo de determinar se o modelo era viável, o modelo hipotetizado foi testado na amostra total, com o grupo com e sem doença crónica combinados $(N=453)$. Para ambos os grupos, o ajuste do modelo foi analisado separadamente. Seguidamente, foi estimado o modelo não restrito (i.e., modelo em que os parâmetros podem variar entre os grupos) e as diferenças nas paths significativas e não significativas entre os dois grupos em comparação foram analisadas. $O$ modelo totalmente restrito (i.e., modelo em que os parâmetros são constrangidos para serem iguais entre os grupos) foi, igualmente, analisado. Estes dois modelos, não restrito e totalmente restrito foram comparados através do teste de diferença do qui-quadrado. Por último, foram calculadas as diferenças no rácio crítico $(z)$, no sentido de se verificar a significância estatística das diferenças entre ambos os grupos (Byrne, 2010; Kline, 2005; Tabachnick \& Fidell, 2007).

\section{Resultados}

\section{Estatística Descritiva e de Correlações}

Os resultados dos testes $t$ revelaram diferenças significativas entre os grupos (grupo com e sem diagnóstico de doença física crónica) em relação à vergonha, ação comprometida e sintomatologia depressiva. O grupo com doença crónica apresentou níveis mais elevados de vergonha e sintomatologia depressiva e menores níveis de ação comprometida, comparativamente ao grupo sem doença crónica. Por outro lado, em relação à autocompaixão não foram encontradas diferenças significativas entre os grupos (Tabela 2).

\section{Tabela 2}

Alfas de Cronbach ( $\alpha$ ), Médias (M) e Desvio-Padrão (DP) e Diferenças (t de Student) das Variáveis em Estudo por Grupo de Participantes com Doença Crónica $(n=223)$ e Grupo sem Diagnóstico de Doença Crónica $(n=230)$

\begin{tabular}{|c|c|c|c|c|c|c|c|c|c|}
\hline & \multicolumn{3}{|c|}{ Grupo com doença crónica } & \multicolumn{3}{|c|}{ Grupo sem doença crónica } & \multirow{2}{*}{$t$} & \multirow{2}{*}{$g l$} & \multirow{2}{*}{$p$} \\
\hline & $\alpha$ & $M$ & $D P$ & $\alpha$ & M & $D P$ & & & \\
\hline EISS & 0,90 & 10,53 & 6,39 & 0,89 & 8,58 & 5,50 & $-3,47$ & 436,94 & 0,001 \\
\hline SELF-AC & 0,89 & 3,19 & 0,67 & 0,92 & 3,24 & 0,71 & 0,69 & 451 & 0,491 \\
\hline CAQ-8 & 0,80 & 30,35 & 8,02 & 0,81 & 32,28 & 7,43 & 2,65 & 451 & 0,008 \\
\hline DASS-21-DEP & 0,92 & 6,03 & 5,79 & 0,93 & 4,34 & 5,41 & $-3,20$ & 446,55 & 0,001 \\
\hline
\end{tabular}

Nota. EISS = Escala de Vergonha Externa e Interna; SELFC-AC = Escala de Autocompaixão [dimensão autocompaixão (AC)]; CAQ-8 = Questionário de Ação Comprometida; DASS-21-DEP = Escala de Ansiedade, Depressão e Stress [Dimensão depressão (DEP)]. $M=M e ́ d i a ;$ $D P=$ Desvio-Padrão; $t=$ Teste $t$ de Student; $g l=$ Graus de liberdade; $p=$ Nível de significância.

${ }^{*} p<0,05 ;{ }^{* *} p<0,01 ;{ }^{* * *} p<0,001$. 
Os resultados das análises de correlação de Pearson (Tabela 3) permitiram observar que, para ambos os grupos (com e sem doença física crónica), tanto o género como a idade não apresentaram associações significativas com nenhuma das variáveis em estudo, exceto a relação entre idade e autocompaixão, no grupo com doença crónica, com a qual revelou uma associação positiva e fraca. A vergonha associou-se negativamente com a autocompaixão e com a ação comprometida, em ambos os grupos, com magnitudes de moderadas a altas. Por outro lado, tanto para o grupo com doença como para o grupo sem doença crónica, a vergonha apresentou uma correlação significativa, positiva e com uma magnitude alta com a depressão. Por sua vez, em ambos os grupos, a autocompaixão revelou-se positiva e moderadamente associada com ação comprometida e negativamente correlacionada com a depressão. Foi ainda possível verificar que a ação comprometida revelou uma associação negativa e alta com a sintomatologia depressiva, tanto no grupo com doença crónica como no grupo sem doença crónica.

\section{Tabela 3}

Correlações de Pearson Entre as Variáveis Estudadas para Grupo com Doença Crónica $(n=223)$ e para Grupo sem Diagnóstico de Doença Crónica ( $n=230)$

\begin{tabular}{|c|c|c|c|c|c|c|c|}
\hline & \multirow[b]{2}{*}{ Medidas } & \multicolumn{6}{|c|}{ Grupo sem diagnóstico de doença crónica } \\
\hline & & Género & Idade & EISS & SELFC-AC & CAQ-8 & DASS-21-DEP \\
\hline \multirow{6}{*}{ 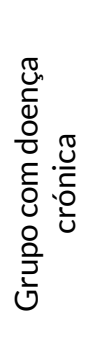 } & Género & - & $-0,09$ & 0,03 & $-0,03$ & $-0,00$ & 0,00 \\
\hline & Idade & $-0,01$ & - & $-0,05$ & 0,07 & 0,13 & 0,01 \\
\hline & EISS & $-0,02$ & $-0,12$ & - & $-0,36^{* * *}$ & $-0,52^{* * *}$ & $0,55^{* * *}$ \\
\hline & SELFC-AC & 0,06 & $0,15^{*}$ & $-0,45^{* * *}$ & - & $0,46^{* * *}$ & $-0,30^{* * *}$ \\
\hline & CAQ-8 & 0,05 & 0,07 & $-0,45^{* * *}$ & $0,37^{* * *}$ & - & $-0,50^{* * *}$ \\
\hline & DASS-21-DEP & $-0,10$ & 0,01 & $0,60^{* * *}$ & $-0,37^{* * *}$ & $-0,50^{* * *}$ & - \\
\hline
\end{tabular}

Nota. EISS = Escala de Vergonha Externa e Interna; SELFC-AC = Escala de Autocompaixão [dimensão autocompaixão (AC)]; CAQ-8 = Questionário de Ação Comprometida; DASS-21-DEP = Escala de Ansiedade, Depressão e Stress [Dimensão depressão (DEP)]. As correlações entre as variáveis estudadas para o Grupo sem diagnóstico de doença crónica estão assinaladas em itálico. ${ }^{*} p<0,05 ;{ }^{* *} p<0,01 ;{ }^{* * *} p<0,001$.

\section{Análise de caminhos (path analysis)}

De forma a compreender as relações existentes entre as variáveis em estudo foi realizada uma path analysis (MacKinnon, 2008), que visou explorar o papel mediador da autocompaixão (SELFC-AC) e da ação comprometida (CAQ-8) na relação entre vergonha (EISS) e sintomatologia depressiva (DASS-21-DEP). O modelo foi inicialmente testado através de um modelo saturado, constituído por 20 parâmetros. Os resultados pareceram indicar a presença de uma path não significativa: o efeito direto da autocompaixão na sintomatologia depressiva ( $b_{\mathrm{SELFC}-\mathrm{AC}}=-0,34 ; S E_{\mathrm{b}}=0,34 ; Z=-1.02 ; p=0,309$ ). Esta path foi eliminada e o modelo foi reajustado e recalculado.

O modelo final, o qual pareceu demonstrar uma excelente adequação aos dados empíricos $\left[\chi^{2}(1)=1.03, p\right.$ $=0,309 ; \mathrm{CFI}=1.00 ; \mathrm{TLI}=1.00 ; \mathrm{RMSEA}=0,01, p=0,537 ; 95 \% \mathrm{IC}=0,00$ a 0,13] (Kline, 2005), explicou 17\% da variância da autocompaixão, $29 \%$ da variância da ação comprometida e $41 \%$ da variância associada à sintomatologia depressiva. 
Os resultados pareceram demonstrar que a vergonha teve um efeito direto na autocompaixão $(\beta=-0,41$; $\left.b_{\mathrm{EISS}}=-0,05 ; S E_{\mathrm{b}}=0,01 ; Z=-9,48 ; p<0,001\right)$, na ação comprometida $\left(\beta=-0,39 ; b_{\mathrm{EISS}}=-0,50 ; S E_{\mathrm{b}}=0,06 ; Z\right.$ $=-8,93 ; p<0,001)$ e na sintomatologia depressiva $\left(\beta=0,45 ; b_{\text {EISS }}=0,42 ; S E_{b}=0,04 ; Z=10,78 ; p<0,001\right)$. A autocompaixão pareceu apresentar um efeito direto na ação comprometida $(\beta=0,26$; bsELFC-AC $=2,89$; $\left.S E_{b}=0,49 ; Z=5,90 ; p<0,001\right)$ a qual, por sua vez, pareceu ter um efeito direto na sintomatologia depressiva $\left(\beta=-0,29 ; b_{\text {CAQ- }-8}=-0,21 ; S E_{b}=0,03 ; Z=-6,97 ; p<0,001\right.$ ).

$A$ análise dos efeitos indiretos pareceu demonstrar que a vergonha apresentava um efeito indireto de 0,10 (95\% IC $=-0,15$ a $-0,07)$ na ação comprometida, o qual pareceu ser mediado através da autocompaixão. Simultaneamente, a vergonha pareceu ter um efeito indireto de 0,14 (95\% IC = 0,09 a $0,20)$ na sintomatologia depressiva, mediado pela autocompaixão e pela ação comprometida. Por sua vez, a autocompaixão pareceu ter um efeito indireto de $-0,07$ (95\% IC $=-0,12$ a $-0,04)$ na sintomatologia depressiva, o qual pareceu ser mediado pela ação comprometida.

Como síntese, os resultados obtidos sugeriram que a vergonha se associou a níveis mais elevados de sintomatologia depressiva e este efeito pareceu ser mediado por menores competências autocompassivas e menos padrões de ação comprometida.

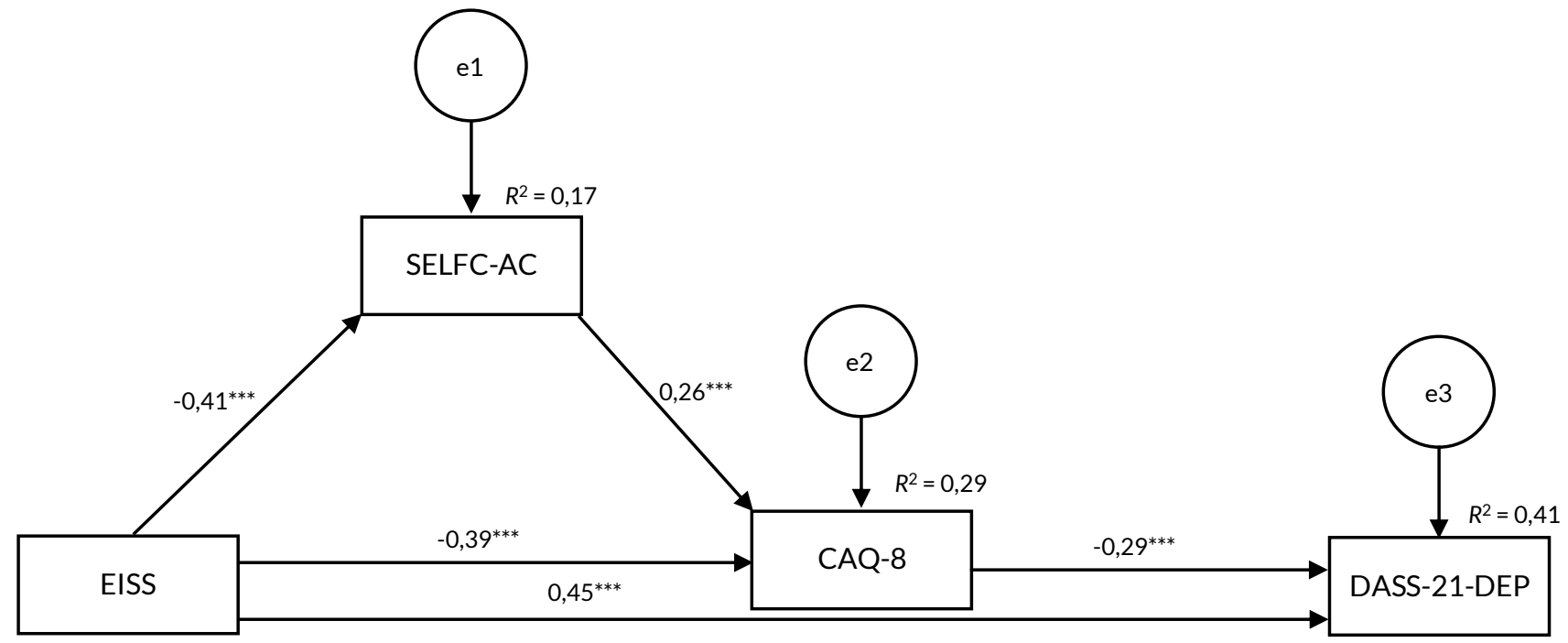

Figura 1. Modelo Final do modelo de Path Analysis. Estão apresentados os coeficientes estandardizados das paths entre as variáveis. EISS = Escala de Vergonha Externa e Interna; SELFC-AC = Escala de Autocompaixão [dimensão autocompaixão (AC)]; CAQ-8 = Questionário de Ação Comprometida; DASS-21-DEP = Escala de Ansiedade, Depressão e Stress [Dimensão depressão (DEP)]. ${ }^{* * *} p<0,001$.

\section{Análise Multigrupos}

De forma a testar a existência de diferenças no modelo entre os dois grupos (grupo com e sem diagnóstico de doença física crónica), foi realizada uma análise multigrupos. Para ambos os grupos o modelo apresentou um ajustamento aos dados empíricos muito bom $\left(\chi^{2}{ }^{2}\right.$ = 1,31, $p=0,521 ; C F I=1,00 ; T L I=1,00 ; R M S E A=0,00,[I C$ $=0,00 ; 0,08] ; p=0,788)$. Os resultados obtidos no teste de diferença do qui-quadrado entre o modelo não restrito (modelo em que os parâmetros podem variar entre os grupos) $\left(\chi^{2}(2)=1,31, p=0,521\right)$ e o modelo totalmente restrito $\left(\chi^{2}(7)=3,89 ; p=0,792\right)$, revelaram a invariância do modelo em relação aos grupos em comparação $\left(\chi^{2 \mathrm{dif}}{ }_{(5)}=2,59 ; p=0,764\right)$. Por fim, para testar as diferenças entre os dois grupos entre todas as 
todas as estimativas de parâmetros, foram calculados os valores de rácio crítico (z). Nenhum coeficiente de parâmetros demonstrou diferenças estatisticamente significativas entre os grupos (valores $z$ entre - 0,85 e 0,80; $p$ > 0,05) (Byrne, 2010; Tabachnick \& Fidell, 2007). Assim, estes resultados pareceram demonstrar que as relações entre as variáveis em estudo não apresentam diferenças nos dois grupos, tanto ao nível do modelo geral como ao nível das paths entre variáveis.

\section{Discussão}

O presente estudo teve como principal objetivo explorar em que medida dois processos psicológicos adaptativos, autocompaixão e ação comprometida, medeiam significativamente o impacto de sentimentos de vergonha na sintomatologia depressiva em pessoas com e sem diagnóstico de doença física crónica. Adicionalmente, estávamos particularmente interessados em explorar diferenças entre os grupos com e sem diagnóstico de doença física crónica, em relação às variáveis em estudo. No que diz respeito às diferenças entre os grupos, os resultados revelaram que o grupo com doença física crónica apresenta pontuações significativamente superiores em relação à vergonha e sintomatologia depressiva, comparativamente ao grupo sem doença física crónica. Estes resultados estão em consonância com o esperado uma vez que os doentes crónicos são teoricamente considerados mais propensos a experienciar sentimentos de vergonha (e.g., na doença inflamatória intestinal; Casati et al., 2000). Contudo, o presente estudo é o primeiro, até ao nosso conhecimento atual, que explora as diferenças nos sentimentos de vergonha em grupos com e sem doença crónica, mostrando que doentes crónicos parecem objetivamente apresentar maiores níveis nesta variável em comparação ao grupo sem diagnóstico de doença física crónica, podendo estes estar associados à experiência de ter doença crónica e sua sintomatologia ou consequências dos tratamentos, acrescentando assim novos dados à literatura. Paralelamente, os níveis de sintomatologia depressiva superiores no grupo com doença crónica estão de acordo com os dados empíricos prévios que suportam que estes indivíduos reportam maiores dificuldades psicológicas (i.e., sintomatologia depressiva) comparativamente a uma amostra de população geral (Pinto-Gouveia et al., 2013). Em consonância com pesquisas anteriores (Pinto-Gouveia et al., 2013), não foram encontradas diferenças significativas entre os participantes com e sem doença crónica em relação aos níveis de autocompaixão. Além disso, o presente estudo revelou diferenças significativas nos níveis de ação comprometida entre participantes com e sem doença crónica. Este dado revela-se particularmente interessante e parece indicar uma menor tendência do grupo com doença crónica em apresentar padrões de ações consistentes com os valores pessoais. Paralelamente este dado pode ser entendido dado a associação já documentada entre vergonha e menor comprometimento com ações congruentes com os valores pessoais (Trindade et al., 2018b). Assim, revelando os indivíduos com diagnóstico de doença crónica maiores níveis de vergonha, é expectável uma menor adoção de comportamentos alinhados com uma vida valorizada.

Os resultados de correlação demonstram que os grupos apresentam um padrão semelhante de associações. Verificou-se que, em ambos os grupos, sentimentos de vergonha estão significativamente associados a menores competências autocompassivas. Este dado está em conformidade com pesquisas anteriores numa amostra de doentes com doença inflamatória intestinal, em relação à vergonha relacionada à doença crónica (Trindade et al., 2017a) e em amostra não clínica, em relação a sentimentos 
gerais de vergonha (e.g., Ferreira et al., 2013). Além disso, como sugerido em estudos prévios que documentam os efeitos adversos da vergonha relacionada à doença crónica (e.g., Trindade et al., 2017a, 2018a) e sentimentos gerais de vergonha (e.g., Kim et al., 2011; Matos \& Pinto-Gouveia, 2010) no funcionamento e bem-estar psicológico, neste estudo a vergonha associou-se positivamente com a sintomatologia depressiva, em ambos os grupos. Contudo, é de salientar que este estudo acrescenta à literatura a relação de uma única medida de experiência de vergonha externa e interna com a sintomatologia depressiva. A ação comprometida apresenta associações negativas relevantes com sintomas psicológicos (i.e., sintomatologia depressiva) e com a vergonha corroborando, assim, resultados anteriores numa amostra da população geral e de mulheres com carcinoma da mama (Trindade et al., 2018b). Estes dados parecem indicar que persistir em ações guiadas pelos valores pessoais se associa a menor vivência de vergonha e de sintomas depressivos.

Adicionalmente, do nosso conhecimento atual este é o primeiro estudo que analisa a relação entre os dois processos psicológicos (i.e., autocompaixão e ação comprometida). Este estudo acrescenta à literatura a associação significativa e positiva entre autocompaixão e ação comprometida. De facto, tanto no grupo com como sem doença física crónica foi verificado que maiores níveis de autocompaixão estão associados a maior adoção de ações congruentes com os valores pessoais. Os resultados também revelaram associações negativas entre autocompaixão e sintomatologia depressiva, o que está de acordo com estudos prévios anteriores que demonstram que níveis mais elevados de autocompaixão estão relacionados a um melhor funcionamento psicológico, em particular, com a diminuição de sintomas depressivos (e.g., MacBeth \& Gumley, 2012; Pinto-Gouveia et al., 2013).

Para explorar a hipótese principal, isto é, se a associação entre vergonha e a sintomatologia depressiva é significativa e mediada pela autocompaixão e ação comprometida, foi realizada uma path analysis. $\mathrm{O}$ modelo teórico testado apresenta uma excelente adequação aos dados, explicando $41 \%$ da variância da sintomatologia depressiva. Os resultados sugerem que parte do efeito que a vergonha tem na sintomatologia depressiva é explicado pelos mecanismos de autocompaixão e ação comprometida. Particularmente, os dados sugerem que a experiência de vergonha se associa a maior sintomatologia depressiva, sendo esta relação parcialmente mediada por menores níveis de autocompaixão e de ação comprometida. Especificamente, foi verificado que a presença de experiência de vergonha está associada a menores níveis de ação comprometida, através de menores níveis de competências autocompassivas. A relação direta encontrada no presente estudo entre vergonha e sintomatologia depressiva suporta dados da literatura existente que demonstram que a vergonha está associada a maior sintomatologia depressiva (e.g., Kim et al., 2011; Pinto-Gouveia \& Matos, 2011; Trindade et al., 2017a, 2018a). Os dados demonstram um efeito direto e negativo da vergonha na autocompaixão e na ação comprometida. De facto, estudos prévios sublinham que indivíduos que experienciam maiores níveis de vergonha apresentam menores níveis de autocompaixão (e.g., Ferreira et al., 2013; Trindade et al., 2017a; Zhang et al., 2018) e de ação comprometida (Trindade et al., 2018b). Foi ainda verificado que permanecer em ações guiadas pelos valores pessoais parece levar a menores níveis de sintomatologia depressiva, o que está em conformidade com estudos existentes (Trindade et al., 2018b). No entanto, este estudo acrescenta dados relevantes à literatura sugerindo que maiores níveis de autocompaixão se associam a um maior envolvimento em ações consistentes com os valores de vida pessoais. Além disso, a ação comprometida revelou-se um importante mediador da associação entre a autocompaixão e sintomas depressivos. 
Experienciar maiores níveis de autocompaixão parece levar a menor sintomatologia depressiva, através dos mecanismos de ação comprometida. É importante ressaltar que os resultados obtidos na análise multigrupos suportam a invariância do modelo testado em participantes com e sem doença física crónica, mostrando que estas relações são semelhantes em ambos os grupos em estudo.

Estes resultados devem ser analisados considerando algumas limitações. Uma das principais limitações relaciona-se com a natureza transversal do estudo, impossibilitando o estabelecimento de conclusões de causalidade entre as variáveis. Outra das limitações relaciona-se com a utilização de questionários de autorresposta, dado que, por traduzir uma maior subjetividade, poderá conduzir a enviesamentos nos resultados. De modo a ultrapassar estas limitações, pesquisas futuras devem, portanto, recorrer a um planeamento longitudinal, assim como a entrevistas para explorar estes dados. O recrutamento dos participantes via online poderá ter causado algum enviesamento na amostragem, limitando a generalização dos dados obtidos. Além disso, o tamanho diminuto da amostra e a proporção desigual de participantes do género feminino e masculino em ambos os grupos constituem uma limitação importante do estudo. Adicionalmente, as amostras em estudo (com e sem diagnóstico de doença crónica) revelaram diferenças significativas entre si quanto às variáveis sociodemográficas (e.g., idade, género e estado civil). Assim, os resultados devem ser replicados em amostras maiores e mais homogéneas em relação ao género e às características sociodemográficas (como idade, estado civil). Adicionalmente, os resultados devem ser explorados em amostras com condições crónicas específicas devido a possíveis especificidades de determinadas doenças, recrutando doentes em centros médicos. Dada a natureza multideterminada e complexa da sintomatologia depressiva, outros processos de regulação emocional não explorados no modelo testado podem estar envolvidos. Desta forma, estudos futuros devem incluir outros processos de regulação emocional de forma a explorar o impacto de outros mecanismos na relação entre a vergonha e sintomatologia depressiva.

Em suma, os dados obtidos permitem corroborar a importância da autocompaixão e ação comprometida enquanto processos mediadores significativos para a explicação da sintomatologia depressiva. Em termos da investigação e da prática clínica, este estudo sustenta a relevância, para a saúde mental, dos processos de autocompaixão e ação comprometida tanto em pessoas sem como com doença física crónica. Suporta, ainda, que intervenções clínicas com o objetivo de melhorar a saúde mental poderão beneficiar da promoção destes processos. De facto, este estudo reforça a pertinência do desenvolvimento de programas de intervenção baseados na compaixão e na ação comprometida, à luz da Terapia Focada na Compaixão e da Terapia de Aceitação e Compromisso, para a melhoria do bem-estar e saúde mental em indivíduos com e sem condições crónicas físicas de saúde.

Conflito de interesses | Conflict of interest: nenhum | none.

Fontes de financiamento | Funding sources: nenhuma | none.

Contributos: DV: Recrutamento e avaliação dos participantes; Pesquisa bibliográfica; Análise estatística; Escrita do manuscrito ao longo de suas fases de desenvolvimento. IT: Desenho do estudo, preparação das medidas e escrita do protocolo; Fornecimento dos resumos de pesquisas anteriores; Análise estatística; Supervisão da escrita do manuscrito ao longo das suas fases de desenvolvimento e aprovação do manuscrito final. CF: Desenho do estudo, preparação das medidas e escrita do protocolo; Fornecimento dos resumos de pesquisas anteriores; Análise estatística; Supervisão da escrita do manuscrito ao longo das suas fases de desenvolvimento e aprovação do manuscrito final. 


\section{Referências}

Arbuckle, J. L. (2013). IBM SPSS Amos 22: User's Guide. Chicago, IL: IBM SPSS.

Benda, J., Kadlečík, P., \& Loskotová, M. (2018). Differences in self-compassion and shame in patients with anxiety disorders, patients with depressive disorders and healthy controls. Československá psychologie, 62(6), 529541. Retrieved from https://eds.b.ebscohost.com/eds/pdfviewer/pdfviewer?vid=0\&sid=cd1dc0cd-402b4187-b6a0-ea1e77a0612e\%40pdc-v-sessmgr05

Byrne, B. M. (2010). Structural equation modeling with AMOS: Basic concepts, applications, and programming (2nd ed.). New York, NY: Routledge.

Casati, J., Toner, B. B., De Rooy, E. C., Drossman, D. A., \& Maunder, R. G. (2000). Concerns of patients with inflammatory bowel disease: A review of emerging themes. Digestive Diseases and Sciences, 45(1), 26-31. https://doi.org/10.1023/a:1005492806777

Castilho, P., \& Pinto-Gouveia, J. (2011). Auto-compaixão: Estudo da validação da versão portuguesa da Escala da Auto-Compaixão e da sua relação com as experiências adversas na infância, a comparação social e a psicopatologia [Self-compassion: Study of the validation of the Portuguese version of the self-compassion scale and its relationship with adverse childhood experiences, social comparison and psychopathology]. Psychologica, 2011(54), 203-230. http://doi.org/10.14195/1647-8606_54_8

Cohen, J., Cohen, P., West, S. G., \& Aiken, L. S. (2003). Applied multiple regression/correlation analysis for the behavioral sciences (3rd ed.). Mahwah, NJ: Lawrence Erlbaum Associates.

Costa, J., \& Pinto-Gouveia, J. (2011). Acceptance of pain, self-compassion and psychopathology: Using the chronic pain acceptance questionnaire to identify patients' subgroups. Clinical Psychology and Psychotherapy, 18(4), 292-302. https://doi.org/10.1002/cpp.718

Coutinho, M., Trindade, I. A., \& Ferreira, C. (2019). Experiential avoidance, committed action, and quality of life: Differences between college students with chronic illness and their healthy peers. Manuscript accepted for publication.

Ferreira, C., Moura-Ramos, M., Matos, M., \& Galhardo, A. (2018). A new measure to assess external and internal shame: Development, factor structure and psychometric properties of the external and internal shame scale. Manuscript submitted for publication.

Ferreira, C., Pinto-Gouveia, J., \& Duarte, C. (2013). Self-compassion in the face of shame and body image dissatisfaction: Implications for eating disorders. Eating Behaviors, 14(2), 207-210. https://doi.org/10.1016/j.eatbeh.2013.01.005

Folkman, S., \& Moskowitz, J. T. (2000). Stress, positive emotion, and coping. Current Directions in Psychological Science, 9(4), 115-118. https://doi.org/10.1111/1467-8721.00073

Galán, S., Roy, R., Solé, E., Racine, M., Vega, R., Jensen, M. P., \& Miró, J. (2019). Committed action, disability and perceived health in individuals with fibromyalgia. Behavioral Medicine, 45(1), 62-69. https://doi.org/10.1080/08964289.2018.1467370

Gilbert, P. (1998). What is shame? Some core issues and controversies. In P. Gilbert \& B. Andrews (Eds.), Shame: Interpersonal behavior, psychopathology and culture (pp. 3-38). New York: Oxford University Press.

Gilbert, P. (2000). The relationship of shame, social anxiety and depression: The role of the evaluation of social rank. Clinical Psychology and Psychotherapy, 7(3), 174-189. https://doi:10.1002/1099-0879(200007)7:3<174::aidcpp236>3.0.co;2-u

Gilbert, P. (2002). Body shame: A biopsychosocial conceptualization and overview, with treatment implications. In P. Gilbert \& Miles (Eds.), Body shame: Conceptualisation, research and treatment (pp. 3-54). London: Brunner.

Gilbert, P. (2007). The evolution of shame as a marker for relationship security: A biopsychosocial approach. In J. L. Tracy, R. W. Robins, \& J. P. Tangney (Eds.), The self-conscious emotions: Theory and research (pp. 283-309). New York: Guilford Press.

Gilbert, P., \& Irons, C. (2005). Focused therapies and compassionate mind training for shame and self-attacking. In P. Gilbert (Ed.), Compassion: Conceptualisations, research and use in psychotherapy (pp. 263-325). London: Routledge.

Gilbert, P., \& Irons, C. (2009). Shame, self-criticism, and self-compassion in adolescence. In N. B. Allen \& L. B. Sheeber (Eds.), Adolescent emotional development and the emergence of depressive disorders (pp. 195-214). London: Cambridge University Press. 
Gilbert, P., \& Procter, S. (2006). Compassionate mind training for people with high shame and self-criticism: Overview and pilot study of a group therapy approach. Clinical Psychology and Psychotherapy, 13(6), 353-379. https://doi.org/10.1002/cpp.507

Hayes, S. C., Luoma, J. B., Bond, F. W., Masuda, A., \& Lillis, J. (2006). Acceptance and commitment therapy: Model, processes and outcomes. Behaviour Research and Therapy, 44(1), 1-25. https://doi.org/10.1016/j.brat.2005.06.006

Hayes, S. C., Pistorello, J., \& Levin, M. E. (2012). Acceptance and commitment therapy as a unified model of behavior change. The Counseling Psychologist, 40(7), 976-1002. https://doi.org/10.1177/0011000012460836

Hu, L.-T., \& Bentler, P. M. (1999). Cutoff criteria for fit indexes in covariance structure analysis: Conventional criteria versus new alternatives. Structural Equation Modeling: A Multidisciplinary Journal, 6(1), 1-55. https://doi.org/10.1080/10705519909540118

Kim, S., Thibodeau, R., \& Jorgensen, R. S. (2011). Shame, guilt, and depressive symptoms: A meta-analytic review. Psychological Bulletin, 137(1), 68-96. https://doi.org/10.1037/a0021466

Kline, R. B. (2005). Principles and practice of structural equation modelling (3rd ed.). New York, NY: Guilford Press.

Krieger, T., Altenstein, D., Baettig, I., Doering, N., \& Holtforth, M. G. (2013). Self-compassion in depression: Associations with depressive symptoms, rumination, and avoidance in depressed outpatients. Behavior Therapy, 44(3), 501-513. https://doi.org/10.1016/j.beth.2013.04.004

Körner, A., Coroiu, A., Copeland, L., Gomez-Garibello, C., Albani, C., Zenger, M., \& Brähler, E. (2015). The role of self-compassion in buffering symptoms of depression in the general population. PLoS ONE, 10(10), e0136598. https://doi.org/10.1371/journal.pone.0136598

Lovibond, P. F., \& Lovibond, S. H. (1995). The structure of negative emotional states: Comparison of the Depression Anxiety Stress Scales (DASS) with the Beck Depression and Anxiety Inventories. Behaviour Research and Therapy, 33(3), 335-343. https://doi.org/10.1016/0005-7967(94)00075-u

MacBeth A., \& Gumley, A. (2012). Exploring compassion: A meta-analysis of the association between selfcompassion and psychopathology. Clinical Psychology Review, 32(6), 545-552. https://doi.org/10.1016/j.cpr.2012.06.003

MacKinnon, D. P. (2008). Introduction to statistical mediation analysis. Mahwah, NJ: Lawrence Erlbaum Associates.

Matos, M., \& Pinto-Gouveia, J. (2010). Shame as a traumatic memory. Clinical Psychology and Psychotherapy, 17(4), 229-312. https://doi.org/10.1002/cpp.659

McCracken, L. M. (2013). Committed action: An application of the psychological flexibility model to activity patterns in chronic pain. The Journal of Pain, 14(8), 828-835. https://doi.org/10.1016/j.jpain.2013.02.009

McCracken, L. M., Chilcot, J., \& Norton, S. (2014). Further development in the assessment of psychological flexibility: A shortened Committed Action Questionnaire (CAQ-8). European Journal of Pain, 19(5), 677-685. https://doi.org/10.1002/ejp.589

Michelson, S. E., Lee, J. K., Orsillo, S. M., \& Roemer, L. (2011). The role of values-consistent behavior in generalized anxiety disorder. Depression and Anxiety, 28(5), 358-366. https://doi.org/10.1002/da.20793

Neff, K. D. (2003a). Self-compassion: An alternative conceptualization of a healthy attitude toward oneself. Self and Identity, 2(2), 85-101. https://doi.org/10.1080/15298860309032

Neff, K. D. (2003b). The development and validation of a scale to measure self-compassion. Self and Identity, 2(3), 223-250. https://doi.org/10.1080/15298860309027

Neff, K. D. (2009). The role of self-compassion in development: A healthier way to relate to oneself. Human Development, 52(4), 211-214. https://doi.org/10.1159/000215071

Pais-Ribeiro, J. L., Honrado, A., \& Leal, I. (2004). Contribuição para o estudo da adaptação portuguesa das Escalas de Ansiedade, Depressão e Stress (EADS) de 21 itens de Lovibond e Lovibond [Contribution to the study of the Portuguese adaptation of the Anxiety, Depression and Stress Scales (EADS) of 21 Lovibond and Lovibond items]. Psicologia, Saúde \& Doenças, 5(2), 229-239. Retrieved from http://scielo.mec.pt/pdf/psd/v5n2/v5n2a07.pdf

Pinto-Gouveia, J., Duarte, C., Matos, M., \& Fráguas, S. (2013). The protective role of self-compassion in relation to psychopathology symptoms and quality of life in chronic and in cancer patients. Clinical Psychology \& Psychotherapy, 21(4), 311-323. https://doi.org/10.1002/cpp.1838

Pinto-Gouveia, J., \& Matos, M. (2011). Can shame memories become a key to identify? The centrality of shame memories predicts psychopathology. Applied Cognitive Psychology, 25(2), 281-290. https://doi.org/10.1002/acp.1689 
Sirois, F. M., \& Hirsch, J. K. (2019). Self-compassion and adherence in five medical samples: The role of stress. Mindfulness, 10(1), 46-54. https://doi.org/10.1007/s12671-018-0945-9

Sirois, F. M., \& Rowse, G. (2016). The role of self-compassion in chronic illness care. Journal of Clinical Outcomes Management, 23(11), 521-527. Retrieved from https://self-compassion.org/wpcontent/uploads/2018/05/Sirois2016.pdf

Tabachnick, B. G., \& Fidell, L. S. (2007). Using multivariate statistics (5th ed.). Boston, MA: Pearson Education.

Trindade, I. A., Duarte, J., Ferreira, C., Coutinho, M., \& Pinto-Gouveia, J. (2018). The impact of illness-related shame on psychological health and social relationships: Testing a mediational model in students with chronic illness. Clinical Psychology and Psychotherapy, 25(3), 408-414. https://doi.org/10.1002/cpp.2175

Trindade, I. A., Ferreira, C., \& Pinto-Gouveia, J. (2017a). Chronic illness-related shame: Development of a new scale and novel approach for IBD patients' depressive symptomatology. Clinical Psychology \& Psychotherapy, 24(1), 255-263. https://doi.org/10.1002/cpp.2035

Trindade, I. A., Ferreira, C., \& Pinto-Gouveia, J. (2017b). Shame and emotion regulation in inflammatory bowel disease: Effects on psychosocial functioning. Journal of Health Psychology, 135910531771892. https://doi.org/10.1177/1359105317718925

Trindade, I. A., Marta-Simões, J., Ferreira, C., \& Pinto-Gouveia, J. (2018a). Chronic illness-related cognitive fusion explains the impact of body dissatisfaction and shame on depression symptoms in breast cancer patients. Clinical Psychology and Psychotherapy, 25(6), 886-893. https://doi.org/10.1002/cpp.2323

Trindade, I. A., Marta-Simões, J., Ferreira, C., \& Pinto-Gouveia, J. (2018b). Developments on committed action: Validity of the CAQ-8 and analysis of committed action's role in depressive symptomatology in breast cancer patients and healthy individuals. Clinical Psychology and Psychotherapy, 25(1), 42-50. https://doi.org/10.1002/cpp.2125

Trompetter, H. R., Klooster, P. M., Schreurs, K. M. G., Fledderus, M., Westerhof, G. J., \& Bohlmeijer, E. T. (2013). Measuring values and committed action with the engaged living scale (ELS): Psychometric evaluation in a nonclinical sample and a chronic pain sample. Psychological Assessment, 25(4), 1235-1246. https://doi.org/10.1037/a0033813

Zhang, H., Carr, E. R., Garcia-Williams, A. G., Siegelman, A. E., Berke, D., Niles-Carnes, L. V., ... \& Kaslow, N. J. (2018). Shame and depressive symptoms: Self-compassion and contingent self-worth as mediators? Journal of Clinical Psychology in Medical Settings, 25(4), 408-419. https://doi.org/10.1007/s10880-018-9548-9 


\section{Anexo A}

Lista de Doenças Crónicas Reportadas pelos Participantes ( $n=223)$ (continua)

\begin{tabular}{|c|c|c|c|}
\hline Doenças & & $n$ & $\%$ \\
\hline \multirow{23}{*}{$\begin{array}{l}\text { Reumatológicas e } \\
\text { musculoesqueléticas }\end{array}$} & Lúpus & 75 & 33,63 \\
\hline & Fibromialgia & 24 & 10,76 \\
\hline & Síndrome de Sjogren & 14 & 6.28 \\
\hline & Artrite reumatóide & 13 & 5,82 \\
\hline & Doença de Behçet & 8 & 3,59 \\
\hline & Espondilite anquilosante & 3 & 1,34 \\
\hline & Osteoartrose & 3 & 1,34 \\
\hline & Artrite idiopática juvenil & 2 & 0,90 \\
\hline & Esclerose sistémica & 2 & 0,90 \\
\hline & Síndrome de Raynaud & 2 & 0,90 \\
\hline & Síndrome do anticorpo antifosfolípidico & 2 & 0,90 \\
\hline & Tendinite & 2 & 0,90 \\
\hline & Vasculites & 2 & 0,90 \\
\hline & Doença articular congénita & 1 & 0,45 \\
\hline & Encefalomielite miálgica & 1 & 0,45 \\
\hline & Epicondilites & 1 & 0,45 \\
\hline & Esclerodermia & 1 & 0,45 \\
\hline & Espondilartropatia & 1 & 0,45 \\
\hline & Espondiloartrite indiferenciada & 1 & 0,45 \\
\hline & Polimiosite & 1 & 0,45 \\
\hline & Rizartrose & 1 & 0,45 \\
\hline & Síndrome de Crest & 1 & 0,45 \\
\hline & Tendinose & 1 & 0,45 \\
\hline \multirow{9}{*}{ Respiratórias } & Asma & 17 & 7,62 \\
\hline & Rinite alérgica & 6 & 2,69 \\
\hline & Bronquite asmática alérgica & 2 & 0,90 \\
\hline & Sinusite & 2 & 0,90 \\
\hline & Asma alérgica & 1 & 0,45 \\
\hline & Bronquite & 1 & 0,45 \\
\hline & Bronquite asmática & 1 & 0,45 \\
\hline & Doença pulmonar obstrutiva crónica & 1 & 0,45 \\
\hline & Enfisema pulmonar & 1 & 0,45 \\
\hline \multirow{10}{*}{$\begin{array}{l}\text { Endócrinas e } \\
\text { hematológicas }\end{array}$} & Diabetes mellitus & 4,93 & 4,93 \\
\hline & Hipotiroidismo & 2,69 & 2,69 \\
\hline & Tiroidite de Hashimoto & 1,34 & 1,34 \\
\hline & Gamapatia monoclonal & 0,45 & 0,45 \\
\hline & Hipercolesterolemia & 0,45 & 0,45 \\
\hline & Hipertiroidismo & 0,45 & 0,45 \\
\hline & PolicitemiaVera & 0,45 & 0,45 \\
\hline & Talassemia & 0,45 & 0,45 \\
\hline & Trombocitemia essencial & 0,45 & 0,45 \\
\hline & Trombofilia & 0,45 & 0,45 \\
\hline
\end{tabular}


Anexo A

Lista de Doenças Crónicas Reportadas pelos Participantes ( $\mathrm{n}=223$ ) (continuação)

\begin{tabular}{|c|c|c|c|}
\hline Doenças & & $n$ & $\%$ \\
\hline \multirow{2}{*}{ Ginecológicas } & Endometriose & 22 & 9,87 \\
\hline & Adenomiose & 1 & 0,45 \\
\hline \multirow{5}{*}{ Renais } & Doença renal crónica & 13 & 5,83 \\
\hline & Doença renal poliquística & 2 & 0,90 \\
\hline & Nefrite & 1 & 0,45 \\
\hline & Osteodistrofia renal & 1 & 0,45 \\
\hline & Síndrome de Alport & 1 & 0,45 \\
\hline \multirow{8}{*}{ Gastrointestinais } & Doença de Crohn & 6 & 2,69 \\
\hline & Doença celíaca & 3 & 1,34 \\
\hline & Colite ulcerosa & 2 & 0,90 \\
\hline & Gastrite crónica & 2 & 0,90 \\
\hline & Colangite autoimune & 1 & 0,45 \\
\hline & Fibrose quística & 1 & 0,45 \\
\hline & Hepatite & 1 & 0,45 \\
\hline & Síndrome do intestino irritável & 1 & 0,45 \\
\hline \multirow{6}{*}{ Dermatológicas } & Psoríase & 7 & 3,14 \\
\hline & Dermatite atópica & 1 & 0,45 \\
\hline & Doença de Hailey-Hailey & 1 & 0,45 \\
\hline & Eczema & 1 & 0,45 \\
\hline & Urticária & 1 & 0,45 \\
\hline & Vitiligo & 1 & 0,45 \\
\hline \multirow{3}{*}{ Cardiovasculares } & Hipertensão arterial & 7 & 3,14 \\
\hline & Insuficiência cardíaca & 1 & 0,45 \\
\hline & Síndrome de Wolff-Parkinson-White & 1 & 0,45 \\
\hline \multirow{3}{*}{ Neoplásicas } & Cancro da mama & 1 & 0,45 \\
\hline & Leucemia mieloide crónica & 1 & 0,45 \\
\hline & Outras neoplasias & 1 & 0,45 \\
\hline \multirow{2}{*}{ Neurológicas } & Dor neuropática & 1 & 0,45 \\
\hline & Esclerose múltipla & 1 & 0,45 \\
\hline \multirow[t]{2}{*}{ Neuromusculares } & Hipertermia maligna & 1 & 0,45 \\
\hline & Miastenia gravis & 1 & 0,45 \\
\hline Oftalmológicas & Glaucoma & 1 & 0,45 \\
\hline
\end{tabular}

